\section{Mini-Review}

Correspondence

Ken F. Jarrell

jarrellk@post.queensu.ca

\title{
Archaeal signal peptidases
}

\author{
Sandy Y. M. Ng, Bonnie Chaban, David J. VanDyke and Ken F. Jarrell \\ Department of Microbiology and Immunology, Queen's University, Kingston, ON K7L 3N6, \\ Canada
}

\begin{abstract}
Signal peptidases are vital enzymes in the protein secretion pathway. In Archaea, type I signal peptidase, responsible for the cleavage of secretory signal peptides from the majority of secreted proteins, and prepilin peptidase-like signal peptidase, responsible for processing signal peptides from prepilin-like proteins like the preflagellins and various sugar-binding proteins, have been identified. In addition, the archaeal signal peptide peptidase, responsible for degradation of signal peptides after their removal from precursor proteins, has been characterized. These enzymes seem to have a mosaic of eukaryal and bacterial characteristics, and also possess unique archaeal traits. In this review, the most current knowledge with regard to these enzymes is summarized, including their cellular function, catalytic mechanism and distribution and conservation among archaeal species. Comparisons are drawn of these enzymes to their bacterial and eukaryal counterparts, and unique archaeal features highlighted.
\end{abstract}

\section{Introduction}

The Archaea constitutes a third domain of life, distinct from the Bacteria and the Eukarya (Woese et al., 1990). Originally thought to inhabit only extreme environments, archaeal species have since been found in a wide variety of diverse habitats, where they play important roles in the ecosystem (Chaban et al., 2006). Many characteristics of archaea often display an amalgam of bacterial, eukaryal and archaeal-specific features. This is apparently the case for the signal peptidases required for processing secreted proteins in archaea.

A functional conservation of the vital cellular processes of protein trafficking and secretion has been observed throughout all three domains of life (Pohlschroder et al., 1997, 2005; Albers et al., 2006). In particular, many proteins are synthesized as preproteins, with N-terminal signal peptides acting as sorting signals for recognition and targeting. These peptides are recognized and cleaved by their corresponding signal peptidases. Archaeal protein secretion is a relatively new research field. Current knowledge comes from identification, cloning and biochemical characterization of individual signal peptidases, as well as biochemical analysis of known secreted proteins and in silico examination of sequenced genomes. Within the last 5 years, examples of archaeal signal peptidase I (SPI), type IV prepilin peptidase (TFPP)-like enzyme and signal peptide peptidase (SPP) have been characterized, greatly extending our knowledge of protein processing and secretion in this domain. Archaeal SPI, TFPP-like and SPP candidates compiled from analyses of sequenced genomes or focused studies are presented in Table 1.

\section{Signal peptides}

Current knowledge on archaeal signal peptides comes mainly from examination of available genomes, since few studies are available on individual secreted proteins. Various computer programs aimed at identification of signalpeptide-bearing proteins from sequenced archaeal genomes have predicted $8-32 \%$ of the proteomes to possess signal peptides (Bardy et al., 2003; Saleh et al., 2001), falling within three categories (Pohlschroder et al., 2005). Archaeal class I signal peptides are generally 20-30 amino acids in length (Bardy et al., 2003) and contain the classical motif - a basic $\mathrm{n}$-region followed by a hydrophobic core (h-region) and ending with a carboxyl-terminal region (c-region) containing the cleavage site (von Heijne, 1983). As in bacteria and eukarya, the archaeal cleavage site follows the $(-3,-1)$ rule, where the -3 and -1 positions relative to the cleavage site are held by small, neutral amino acids (often alanine). In silico studies that focused on a single archaeon at a time have indicated certain eukaryal-like features of archaeal signal peptides (Albers \& Driessen, 2002; Nielsen et al., 1999). However, a subsequent genomic survey of 13 archaeal species indicated that their class I signal peptides were more like those of bacteria than eukaryotes in overall composition and amino acid preference at key positions around the cleavage site (Bardy et al., 2003). Class I signal peptides are cleaved by SPI, which processes the majority of secreted proteins exported via the Sec system. Also processed by SPI may be substrates of the Tat translocon (used for folded proteins) (Paetzel et al., 2002; Sargent et al., 2006) that possess generally longer signal peptides distinguished by a less hydrophobic h-region and the presence of the twin arginine motif [(S/T)-R-R-X- $\Phi-\Phi$, where $\mathrm{X}$ can be any amino acid and $\Phi$ is any hydrophobic amino acid] (Berks 1996; Pohlschroder et al., 2005). Apparent archaeal class II signal peptides containing a bacterial-like lipobox $(-2[\mathrm{~L} / \mathrm{I} / \mathrm{G} / \mathrm{A}]-1[\mathrm{~A} / \mathrm{G} / \mathrm{S}]+1 \mathrm{C})$ have been detected in numerous archaeal proteins (Albers et al., 2006; Bardy et al., 2003); this is intriguing, given the unresolved issue of whether archaeal SPII homologues exist (see below). Archaeal class III 
Table 1. Distribution of signal peptidases and signal peptide peptidases in Archaea

The GI numbers (protein) listed are from sequenced genomes found at NCBI Genomes (http://www.ncbi.nlm.nih.gov/genomes/static/a.html).

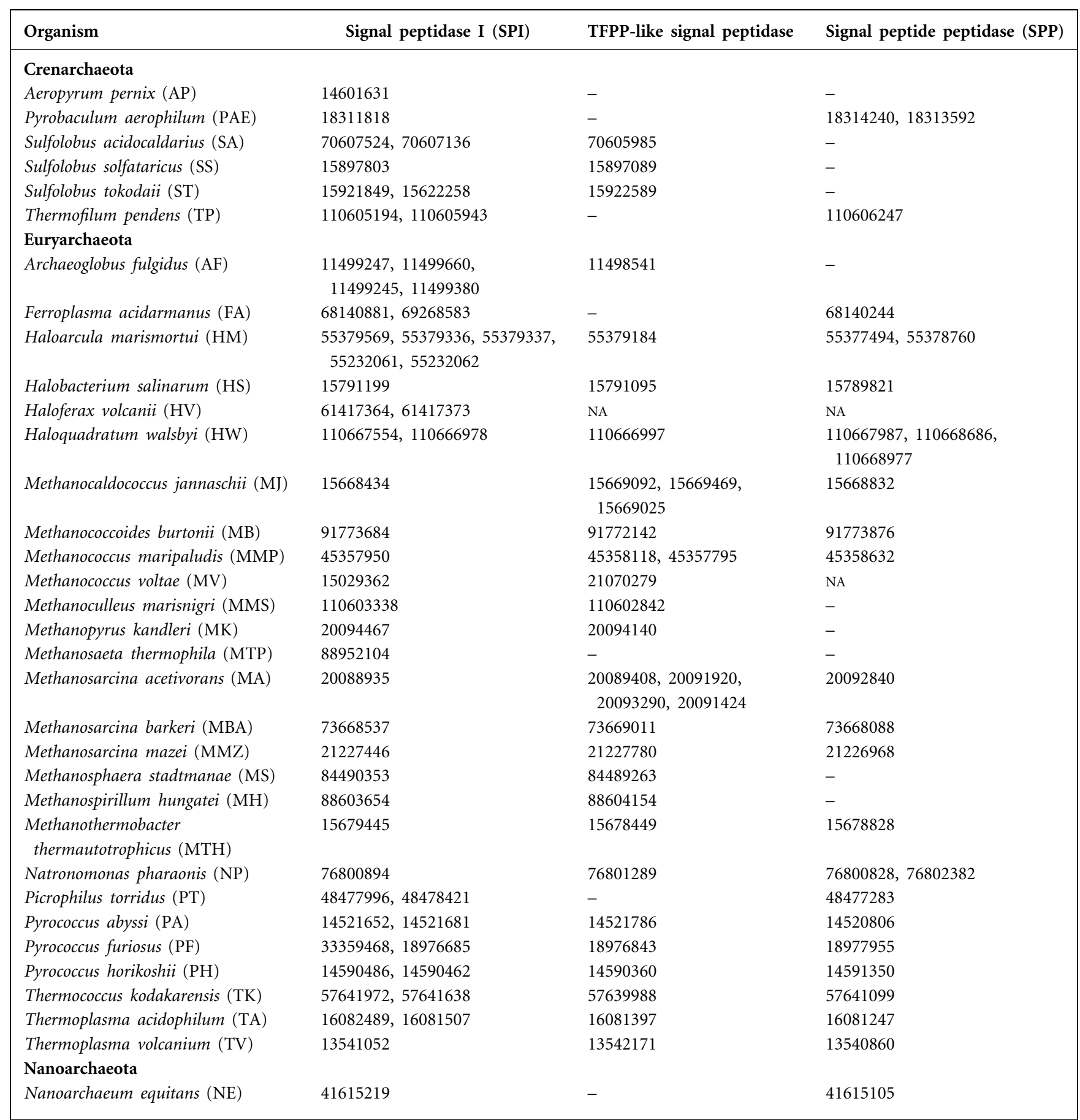

NA, Complete genome sequence is not publicly available for Haloferax volcanii or Methanococcus voltae; the GI numbers reported are from dedicated studies on the enzymes indicated for these organisms.

signal peptides are equivalent to bacterial type IV prepilin signal peptides. These signal peptides are distinct in that their cleavage site is directly after the n-region, leaving the hydrophobic h-region as part of the mature protein. This property is essential to type IV pilin-like proteins for their proper anchoring and assembly into surface structures. In archaea, the preflagellin peptidase processes this class of signal peptides. 


\section{Signal peptidase I (SPI)}

SPI is the essential housekeeping peptidase responsible for processing the majority of preproteins that are transported through either the Sec or the Tat secretion pathway (Sargent et al., 2006; Pohlschroder et al., 2005; Paetzel et al., 2002). While limited sequence identity exists between SPI enzymes, all members of the SPI family possess highly conserved regions that are believed to have important roles in protein function. They are termed boxes A-E, with box A being the membrane-anchoring domain and boxes B-E participating in catalysis. While sequence alignments reveal a universally conserved serine residue within box $\mathrm{B}$, two subgroups exist. The P-type enzyme (found in bacteria, mitochondria and chloroplasts) has a conserved essential lysine while the endoplasmic reticulum (ER)-type enzyme (found in eukarya, archaea and limited bacterial species) has a histidine residue in replacement (Dalbey et al., 1997). In only rare cases, ER-type SPIs have been found in bacteria, with the best-studied example being Bacillus subtilis. This organism has five chromosomally encoded SPIs (Tjalsma et al., 1999) with one, SipW, having a histidine replacement for the conserved lysine found in P-type enzymes. Presumably, SipW shares the catalytic mechanism of ER-type SPIs (Tjalsma et al., 2000). The origin of the ER-type SPI found in bacteria is unclear. Several possibilities have been suggested, including convergent evolution of the P- and ER-type enzymes or a horizontal gene transfer event (Eichler, 2002).

It is generally accepted that P-type SPI enzymes utilize a SerLys catalytic dyad and not the Ser-His-Asp triad commonly found in serine proteases (Dalbey et al., 1997; Tuteja 2005). Site-directed mutagenesis studies in Escherichia coli revealed that $\mathrm{Ser}^{90}$ and Lys ${ }^{145}$ are critical amino residues for catalysis (Tschantz et al., 1993), while subsequent mutagenesis work and analysis of the X-ray structure of the E. coli enzyme identified another residue, $\operatorname{Ser}^{278}$, as essential for optimal activity (Paetzel et al., 2002). The proposed mechanism of catalysis is that $\mathrm{Ser}^{90} \mathrm{O} \gamma$ would perform a nucleophilic attack on the si-face of the scissile bond of the signal peptide, with Lys ${ }^{145} \mathrm{~N} \zeta$ acting as the general base.

The mechanism of action of the ER-type SPI is quite distinct. In the yeast Sec11p subunit of signal peptidase, mutagenesis targeting lysine residues revealed the non-essential nature of all lysines - suggesting that the enzyme might rely on a different catalytic mechanism (VanValkenburgh et al., 1999). Although the exact mechanism of catalysis is unknown, it has been determined through mutagenesis that a conserved serine, histidine and two aspartic acids are important for activity (Dalbey et al., 1997; VanValkenburgh et al., 1999). Furthermore, studies on the ER-type SPI of $B$. subtilis, SipW, identified conserved Ser, His and Asp residues critical for activity, suggesting a Ser-His-Asp catalytic dyad or Ser-His catalytic dyad as potential mechanisms (Tjalsma et al., 2000).

The archaeal SPI enzyme was first identified and biochemically characterized in Methanococcus voltae ( $\mathrm{Ng} \&$ Jarrell,
2003). The putative SPI gene was cloned and overexpressed in E. coli. Enzymic activity was demonstrated through an in vitro peptidase assay. Computer-based topological analyses of $M$. voltae and other archaeal SPI enzymes all indicate the active site to be on the external face of the cytoplasmic membrane. Thus, as in other SPIs, it seems that the archaeal enzyme cleaves the signal peptides from preproteins on the external face of the cytoplasmic membrane, thereby releasing the translocated protein from the membrane. In archaea, the potential processing of signal sequences of integral cytoplasmic membrane proteins has not been well studied. A recent proteomics survey of Natronomonas pharaonis and Halobacterium salinarum indicates that many, if not most, integral membrane proteins lack cleaved signal sequences (Falb et al., 2006). In addition, there are no reports in archaea on the processing of Tat signal peptides by SPI.

In agreement with early insights gained from sequence analysis (Eichler, 2002), archaeal SPI apparently possesses traits from both its bacterial and eukaryal counterparts. Similar to bacterial SPI, archaeal SPI seems to operate independently and not as part of a eukarya-like complex. Genes corresponding to those encoding the subunits of the eukaryal signal peptidase complex have not been identified in genomic searches in archaea, and the overexpressed cloned version of the archaeal enzyme alone is biochemically active (Ng \& Jarrell, 2003). The possibility remains, however, that the archaeal SPI enzyme functions optimally when complexed with additional subunits that are archaeal specific and simply not identified in genome searches (Fine et al., 2006).

Regions with similarities to signature motifs of the SPI family were identified in all the known archaeal SPIs to date (Table 2). The apparent conservation of the catalytic residues in the archaeal SPI with its eukaryal counterpart raised questions as to whether it shared the ER-type SPI proposed mechanism of action. Site-directed mutagenesis was employed targeting the conserved Ser, His and Asp residues of SPI in M. voltae (Bardy et al., 2005). A deviation from Sec11 was noted - while the yeast enzyme has a strict requirement for both conserved aspartic acids, $\mathrm{Asp}^{148}$, but not $\mathrm{Asp}^{142}$, was proven to be essential in M. voltae. On the other hand, the essential nature of $\mathrm{Ser}^{52}$ and $\mathrm{His}^{122}$ was established; in particular, a histidine-to-lysine change led to inactivity of the enzyme, consistent with eukaryal Sec11 mutagenesis results. The mechanism of the peptidase likely involves the ER-type Ser-His-Asp triad. Indeed, almost all archaeal SPIs have the conserved Ser, His and two Asp residues separated by $5-8$ amino acids corresponding to the ones targeted for mutagenesis in M. voltae (Table 2).

Certain archaeal species possess SPI enzymes with unique characteristics that set them apart from the majority of the archaeal SPIs. It was noted upon examination of archaeal SPI sequences that a few (e.g. in Thermoplasma species) contain a bacteria-like domain II region (Eichler, 2002). Domain II is absent in the ER-type SPI; it folds into a large $\beta$ 
Table 2. Partial alignment of archaeal signal peptidase I candidates indicating sequences around catalytically important amino acids

Abbreviations for organisms as in Table 1. ECO, Escherichia coli. BSU, Bacillus subtilis. SC, Saccharomyces cerevisiae. BSU_1731037 is the ER-type SipW enzyme. PF_18976685 is a partial sequence (missing some catalytic boxes) and was not included. Shading key: $\mathbf{\square}, 100 \%$ conservation; , $>80 \%$ conservation; , $>60 \%$ conservation. Conserved substitutions are considered equal; $S=T, V=I=L=M, D=N, K=R$, $\mathrm{F}=\mathrm{Y}=\mathrm{W}$. The proposed catalytic residue in third alignment row is in bold.

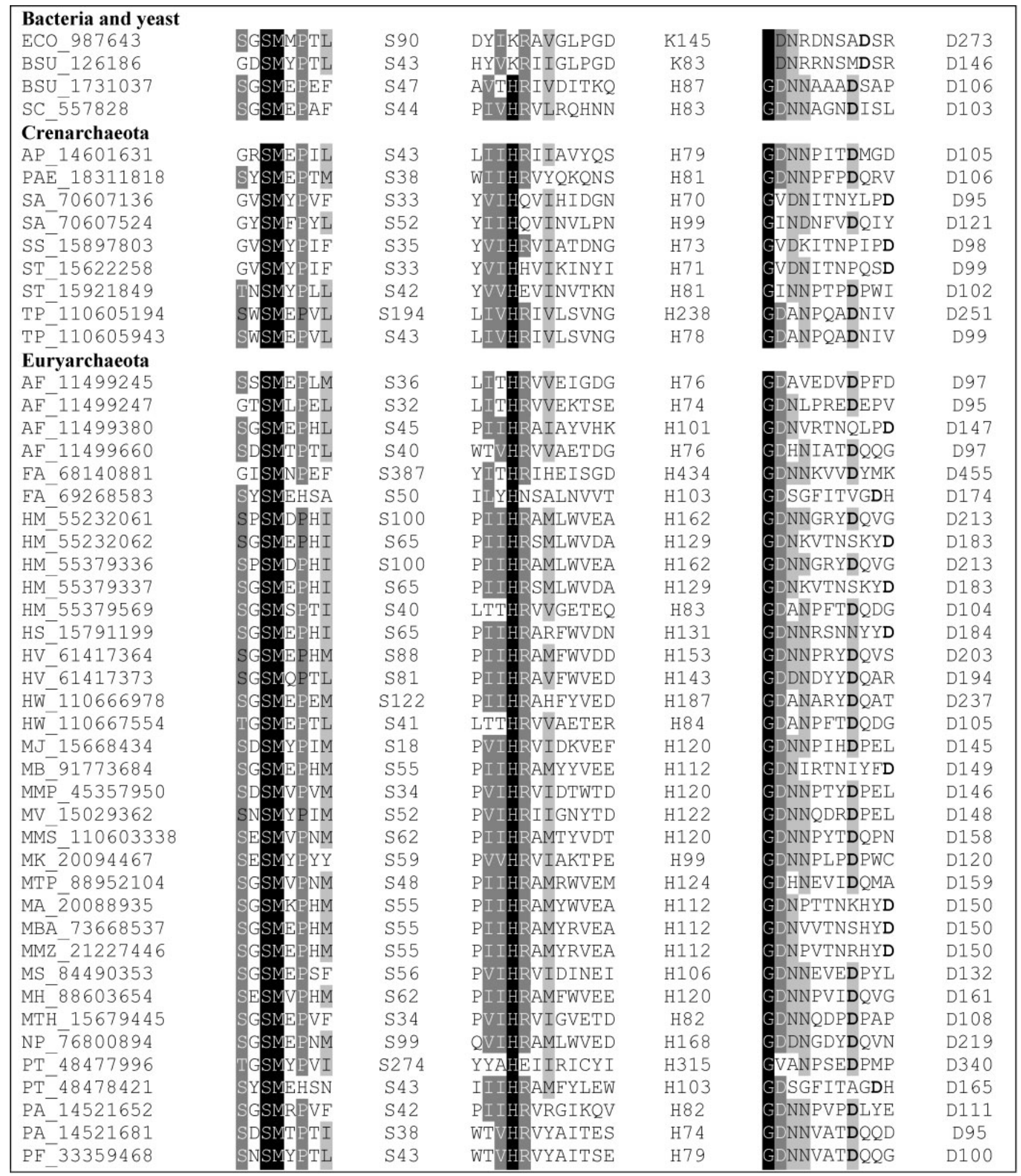


Table 2. cont.

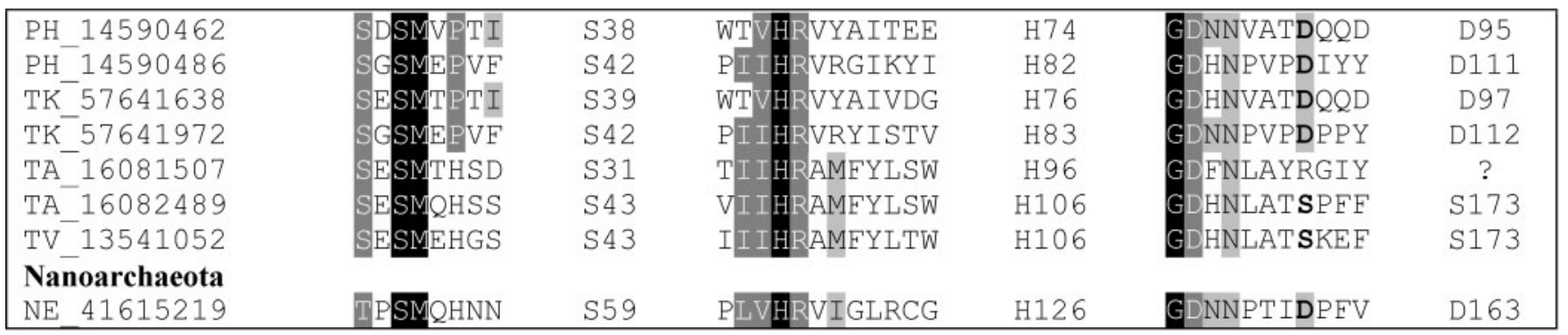

sheet structure on top of the bacterial SPI catalytic core and its function is currently unknown. It has been hypothesized that domain II is a trait in primitive archaeal SPI that has been lost in some species upon diversification (Eichler, 2002). Interestingly, also noted in these SPIs is the absence of the second conserved Asp equivalent. A serine is found in these enzymes at that position, which may be equivalent to the $\operatorname{Ser}^{278}$ shown to be required for optimal activity in E. coli SPI. These unusual archaeal SPIs may have similarities in catalysis to the P-type SPI enzymes.

The identification of paralogous copies of SPI in some archaea (Table 1) is an interesting observation given the high overall similarity of the enzymes and that not all archaea express multiple versions of the enzyme. In many bacteria, such as E. coli, one P-type SPI seems to suffice for the processing of secretory preproteins, although $B$. subtilis possesses five SPI paralogues. The presence of two paralogous ER-type SPIs seems to be characteristic for most eukaryotic species (Dalbey et al., 1997), though the yeast Saccharomyces cerevisiae contains only one. A recent study on Haloferax volcanii (Fine et al., 2006) identified two signal peptidases, Sec1la and Sec11b, both containing conserved boxes A-E, with the conserved Ser and His. Both genes are expressed and both enzymes were active in an in vitro assay using a reporter substrate, although different cleavage efficiencies were observed. Gene deletion studies revealed that only sec11b was essential for viability. Possibilities raised to explain the presence of multiple SPI paralogues in archaea included differential expression in response to growth phase/environmental cues, substrate preferences and perhaps dedication of one of the enzymes to processing substrates that subsequently go through the Tat secretion system, and the possibility that the extra copy just serves as a backup when the amount of signal-peptidebearing substrates increases (Fine et al., 2006). Further experiments are required to evaluate these possibilities and to address the question of why only certain archaea seem to possess multiple paralogues.

\section{Signal peptidase II (SPII)}

Bacterial lipoprotein signal peptides possess conserved n-, $\mathrm{h}$ - and c-regions but also a lipobox in the c-region with the invariant +1 cysteine residue lipid-modified prior to processing by SPII. Signal peptides with such properties have been reported in numerous archaeal proteins (Albers et al., 2006; Bardy et al., 2003). However, no SPII homologues have yet been reported in any annotated archaeal genome, nor were any found by extended searches through BLAST, COG or Pfam. Many potential Tat substrates in archaea also contain a lipobox in their signal peptides (Bolhuis, 2002; Rose et al., 2002; Pohlschroder et al., 2005). Due to the unusual nature of archaeal lipids that may be attached to archaeal lipoproteins, an archaeal SPII equivalent may possess unusual properties rendering them undetectable by current technology. In a recent large-scale proteomics survey of two haloarchaea, Halobacterium salinarum and Natronomonas pharaonis, it was revealed that as many as one-sixth of the archaeal proteins are acetylated. However, these occur via acetylation of the $\mathrm{N}$ terminal residue by $\mathrm{N}$-terminal acetyltransferase, a type of modification that is independent of a lipobox sequence or the cleavage of a signal sequence (Falb et al., 2006).

\section{Prepilin peptidase (TFPP)-like signal peptidases}

Archaeal flagella are composed primarily of flagellins, which are unrelated to their namesakes in the bacterial domain $(\mathrm{Ng}$ et al., 2006). Among several unique features of the archaeal motility apparatus is the presence of signal peptides at the Nterminus of preflagellins. These signal peptides and the Ntermini of mature flagellins bear significant similarity to bacterial type IV pilins, the major structural proteins of type IV pili. Type IV pili are filamentous surface structures in bacteria which allow for surface motility called twitching (Mattick, 2002). These pilins typically have unusually short signal peptides often ending in lysine-glycine, followed by a hydrophobic stretch of $\sim 20$ amino acids. They are processed by a specific signal peptidase, termed the type IV prepilin peptidase (Lory \& Strom, 1997). TFPP is responsible for processing other proteins, called pseudopilins, which have a pilin-like signal peptide and N-terminus. These pseudopilins are thought to represent minor pilins found in the type IV pilus structure as well as other proteins (e.g. PulG) needed to form the 'pseudopilus' in type II secretion systems (Mattick, 2002).

The archaeal TFPP-like enzyme (FlaK, preflagellin peptidase) is responsible for processing flagellins of the euryarchaeotes Methanococcus maripaludis and $M$. voltae (Bardy \& Jarrell, 2002, 2003). The equivalent enzyme in the 
crenarchaeote Sulfolobus solfataricus (PibD) processed not only flagellins but also certain sugar-binding proteins (Albers et al., 2003; Szabo et al., 2006). These sugar-binding proteins may assemble into a filament-like structure ('bindosome'), akin to flagella/pili, that extends from the cell surface (Albers et al., 2006). Furthermore, several other potential substrates of PibD, including some seemingly unrelated to either flagellation or sugar-binding activity, were proposed based on genome analysis of proteins with pilin-like signal peptide cleavage sites and a hydrophobic mature N-terminus (Albers \& Driessen, 2002). Since related Sulfolobus species seem to lack most of these other potential substrates (Albers et al., 2003), and other archaea, such as methanogens, lack sugar-binding proteins, it has been suggested that PibD was unusual among the prepilin peptidase-like enzymes in archaea in having an abnormally large number of substrates ( $\mathrm{Ng}$ et al., 2006). However, subsequently it has been reported that substrates for TFPPlike enzymes may be more numerous than initially suspected throughout the entire archaeal domain (Albers et al., 2006). Although experimental proof is lacking, there are genes in several archaea that could encode type IV pilins (see Albers et al., 2006) that might form the thin filaments observed in electron micrographs of many archaea. It would not be unreasonable to assume that if these represent pilins, they would be processed by the same or closely related signal peptidases that process archaeal flagellins. Indeed, in at least Methanocaldococcus jannaschii and Methanococcus maripaludis, genes that encode prepilin peptidase-like enzymes are found within putative type IV pilin gene clusters (Table 3: M. jannaschii GI:15669025; M. maripaludis GI: 45357795).

Prepilin peptidase-like enzymes are found in most archaea, including both the Euryarchaeota and Crenarchaeota branches (Table 3). Since it is necessary for flagellin processing, this enzyme should be present in all flagellated archaea. Indeed, it is found in all such cases with rare exceptions such as Pyrobaculum. However, since the enzyme can process substrates unrelated to flagellins as in $S$. solfataricus, the enzyme could be found in nonflagellated archaea. This is also true, as attested by a homologue found in Methanothermobacter thermautotrophicus, for instance.

The archaeal TFPP-like enzymes are grouped with bacterial TFPPs in COG1989. Like the bacterial enzyme, the archaeal TFPP-like enzyme is believed to have its active site on the cytoplasmic side of the cytoplasmic membrane (Bardy \& Jarrell, 2003; Szabo et al., 2006). Bacterial TFPPs are usually bifunctional, performing signal peptide cleavage as well as methylation of the subsequent $\mathrm{N}$-terminal amino acid (Lory \& Strom, 1997). In archaea, there is no evidence for Nterminal amino acid modification in processed substrates, indicating that archaeal TFPPs lack methylase activity. Interestingly, a novel subclass of bacterial TFPPs that also lacks methylase activity (exemplified by TadV in Actinobacillus actinomycetemcomitans) (Tomich et al., 2006) has recently been identified.
In both Methanococcus voltae FlaK and S. solfataricus PibD, analysis of key amino acid residues in the substrate necessary for signal peptide processing has been reported. The sequence prior to the cleavage site for the flagellins is highly conserved, with an almost universal presence of glycine at position -1 and with a basic amino acid (lysine or arginine) at position -2. In the case of FlaK (Thomas et al., 2001 ), the -1 glycine and -2 basic amino acid were critical for in vitro preflagellin processing. Among several amino acids tested, only substitution of alanine at -1 allowed processing, albeit poorly, a case mirrored in TFPPs. Similar results were determined for PibD (Albers et al., 2003) although a glycine or alanine at -1 led to similar levels of processing. PibD was less stringent than FlaK in the residues allowed at the -2 position, again supporting the broader substrate specificity of PibD. Requirements of the archaeal TFPP-like enzymes for amino acids at positions downstream of the processing site have not been as well explored. However, in all known archaeal flagellins, the +3 position is occupied by a glycine residue. In the case of M. voltae flagellins, a site-directed change of this glycine to valine resulted in nonprocessing, showing the importance of residues within the mature portion of the protein in the cleavage reaction (Thomas et al., 2001). Inactivation of flaK in $M$. voltae led to nonprocessed flagellins and nonflagellated cells, indicating the necessity of proper flagellin processing in filament assembly (Bardy \& Jarrell, 2003).

TFPPs represent a novel family of aspartic acid proteases (LaPointe \& Taylor, 2000). Site-directed mutagenesis of FlaK and PibD indicated that both aspartic acids residues that aligned with the essential residues for bacterial TFPP activity were also essential in the archaeal enzymes (Bardy \& Jarrell, 2003; Szabo et al., 2006). These two essential aspartic acid residues are found in all archaeal prepilin peptidase-like enzymes (Table 3 ), clearly indicating the same catalytic mechanism for the enzymes from the two prokaryotic domains. Interestingly, the second conserved aspartic acid in archaeal and bacterial TFPPs is found within a motif resembling that found in presenilin aspartic acid proteases $(\gamma$ secretase), i.e. G(A)xGD (Steiner \& Haass, 2000). The importance of the glycine residue immediately preceding the catalytic aspartic acid (Steiner \& Haass, 2000), as well as the X amino acid (Yamasaki et al., 2006), for catalytic function and substrate identification has been demonstrated in the case of the $\gamma$-secretases but no studies have been performed on the corresponding amino acids in the archaeal enzymes.

\section{Signal peptide peptidase (SPP)}

SPPs, found in all three domains of life, are enzymes that cleave signal peptides after their removal from the precursor proteins by signal peptidases. Eukaryotic SPPs are members of the polytopic GxGD aspartic acid proteases that include TFPPs and $\gamma$-secretases (Yamasaki et al., 2006), whose catalytic mechanism is dependent on two aspartate residues. Eukaryotic SPPs are intramembrane enzymes that have been shown to cleave peptide bonds in the plane of the lipid bilayer, unlike conventional proteases with active sites 
Table 3. Partial alignment of archaeal prepilin peptidase-like signal peptidase candidates indicating sequences around catalytically important amino acids

\begin{abstract}
Abbreviations for organisms as in Table 1. ECO, Escherichia coli. PUA, Pseudomonas aeruginosa. Shading key as in Table 2. Numbers indicate the position of the catalytically important aspartic acid residues determined from the GenBank accession number. In some cases there are potential downstream in-frame start sites that yield positions for the catalytic aspartic acids that are more typical. These cases are shown in parentheses.
\end{abstract}

\begin{tabular}{|c|c|c|c|c|}
\hline \multicolumn{5}{|l|}{ Bacteria } \\
\hline ECO_7674168 & SAWLIAASVI & D143 & LRKEALGMGDVLLFAALGGWVG & D208 \\
\hline PUA_15599724 & TWGLIAMSLIDADHQLLPDVLV & D149 & FKLLAMLGAWGG & D213 \\
\hline \multicolumn{5}{|l|}{ Crenarchaeota } \\
\hline SA $70605985 *$ & SIMLAHTSYLDIKKREVDLKIW & D21 & ILSIILSLSNA & D78 \\
\hline SS_-15897089 & IIMLIHTSILDLKYREVDPKIW & D23 & FLNVILSLANA & D80 \\
\hline $\mathrm{ST}^{-} 15922589$ & SIMLIHTSELDLKTREIDLKIW & D27 & YWLSLMGGADLFLSLILSFSNA & D84 \\
\hline \multicolumn{5}{|l|}{ Euryarchaeota } \\
\hline $\mathrm{AF} 11498541$ & LGFLIYACK & D21 & KAIMALAVIFP & D84 \\
\hline HM_55379184† & VPVFGWAAYRDVKTRRVPNRTW & D24 & KAFMLIAVLFP & D93 \\
\hline HS_-15791095* & VPALGWAAVRDIHTRRVSNTLW & D25 & ARAFMVLAVLFP & D92 \\
\hline HW_110666997 & MP ILVWWAWRDVVKTRRLPSYLW & D24 & AKVLMTLAILIP & D96 \\
\hline MJ_15669025 & FILLILAAITDIKERIIPHKYT & D22 & VKLETALAP IFA & D8 4 \\
\hline MJ_15669092 & AIGLIIASIYDLKSREIEDYVW & D22 & KLIMGLGALIP & D83 \\
\hline MJ_15669469 & LICSIYGAVEDWRKREVTDFLW & D19 & KFLMGLSYLKG & D116 \\
\hline MB_91772142 & LLFLIYSCYSDIKTRRVTNKLW & D21 & FYFGAFGGADAKILMVISLILP & D82 \\
\hline MMP_ 45357795 & FLLILTATYTDIKERIIPHFVI & D26 & VKMFTALSPLFA & D88 \\
\hline MMP_45358118 & VIGLILASVQDFRSREIEDYIW & D18 & GKILIGLGALVP & D79 \\
\hline MV_ 21070279 & LLGLIIASIQDIKSREIENYIW & D18 & GKILMGMGALIP & D79 \\
\hline MMS__110602842 & GITLIYASILDAKERRVPHKTW & $\mathrm{D} 29(18)$ & AYALIIITACIP & D93 (82) \\
\hline MK_z̄20094140 & LAVATVAAITDLKWGIVPNRLT & D19 & KLLPSLTLFLH & D76 \\
\hline MA_20089408 & MPELIYACYTDLKARRVSNKVW & D21 & FQLGAFGGGDAKGLIVLSILFP & D82 \\
\hline MA_20091424 & SLFLVLACYTDLRYRRVSNRSC & D18 & FKKGCFGGGDAKSLILISLLFP & D72 \\
\hline MA_20091920 & MAFLIYSCYSDLKARRVSNGVW & $\mathrm{D} 39(21)$ & FHLGAFGGGDAKGLLVLSILFP & $\mathrm{D} 100(82)$ \\
\hline MA_20093290 & LPELIYSSYLDIETRRVPNRIW & D21 & FRLRVEGGADAKALIVIGTLVP & D82 \\
\hline $\mathrm{MH} \_88604154$ & LITLIYGCREDIRERAVPVVMW & D33 (22) & TRFHLMGMADTKALILITVLVP & D98 (87) \\
\hline MBĀ & IPFLIYSCYSDLNSRRVSNKVW & D21 & FQVGAFGGGDAKGLIVLSILFP & D82 \\
\hline MMZ_21227780 & MPELIYGCYTDLKERRVSNKVW & $\mathrm{D} 25(21)$ & FQLGAFGGGDAKGLIVLSILFP & D86 (82) \\
\hline MS_ $\overline{8} 4489263$ & TVCCIIATYTDVKYGIIPNKLT & D25 & WYLGLWAGGDVKLFTAISTLLV & D85 \\
\hline MT $\bar{H} \_15678449$ & LLACFYASYSDIKRGIIPNRLT & $\mathrm{D} 25(22)$ & WRMVAWAGGDVKLFTAVTSLLP & D87 (84) \\
\hline $\mathrm{NP}=\overline{7} 6801289$ & VPAFAWAAYRDIETRRIPNRTW & D53 (26) & WRIGGFGGADAKAIITLAVAFP & D127(100) \\
\hline PA_14521786 & VIVGILTSYTDIKTSYIYEEHF & D18 & YYTGGWASGDVIILGAYSALLP & D105 \\
\hline PF_18976843 & ILVGILTSYTDIKTSYIYEERF & D19 & YYTGGWASGDVLILAAFSSLLP & D106 \\
\hline PH_14590360 & TIVGILTSYTDVKTSYIYEEHF & D18 & YYTGGWASGDVIILGAYSALLP & D105 \\
\hline TK_57639988 & LLTGILTSYTDIKTGEVEDVHA & D20 & YYIGAWASGDVVILAAFSALLP & D111 \\
\hline TA_16081397 & AATAVYSSVSDIKRRSVNSFTF & D27 & FRETLFGIGDVKAETSYLLAFQ & D118 \\
\hline TV 13542171 & LPLLIFGSHSDVKKRSINSFTF & D25 & FGELLFGIGDIKGIVSVVLLFS & D116 \\
\hline
\end{tabular}

${ }^{*}$ SA_70605985 and HS_15791095 as annotated are missing both conserved aspartic acid residues. However, using potential upstream in-frame start sites, both proteins align over their full length with other archaeal enzymes and the two conserved aspartic acid residues are found. These versions are used here.

$\dagger$ †M_55379184 as annotated starts after both conserved aspartic acid residues. We have used the TIGR annotation of this gene (NT01HMA2463), which uses an upstream start codon.

exposed to an aqueous environment (Lemberg \& Martoglio, 2004). In bacteria, only limited data have been published on SPPs - while they have been identified in E. coli and $B$. subtilis as enzymes acting in concert with additional protease(s) to degrade signal peptides (Bolhuis et al., 1999; Novak \& Dev, 1998), their mechanism of action is currently unreported. First insight into the catalytic mechanism of prokaryotic SPPs comes from the enzyme SppA of the hyperthermophilic archaeon Thermococcus kodakaraensis. SppA efficiently cleaved peptides with a relatively small side chain at the $\mathrm{P} 1$ position and a hydrophobic or aromatic residue at the P3 position. The positively charged Arg residue was preferred at the P4 position. Any substrates with an acidic residue at P2, P3 or P4 were not cleaved (Matsumi et al., 2005).

Many of the amino acid residues that are highly conserved amongst the SPPs of bacteria and various archaeal species 
Table 4. Partial alignment of archaeal signal peptide peptidase candidates indicating sequences around catalytically important amino acids

Abbreviations for organisms as in Table 1. BSU, Bacillus subtilis. Shading as in Table 2.

\begin{tabular}{|c|c|c|c|c|}
\hline \multicolumn{5}{|l|}{ Bacteria } \\
\hline BSU_2635437 & GSMAAS & S147 & KDIMSPSREM & K199 \\
\hline \multicolumn{5}{|l|}{ Creñarchaeota } \\
\hline PAE_18313592 & NGLA & S113 & YGRELTDY & K162 \\
\hline PAE 18314240 & AGLAAS & S158 & YGMDLTEF & K207 \\
\hline TP_Ē110606247 & KGLLAS & S125 & ADVGSPRPM & K177 \\
\hline \multicolumn{5}{|l|}{ Euryarchaeota } \\
\hline EA_68140244 & EGIC & S76 & INSPERPM & K128 \\
\hline HM_55377494 & TDVC & S151 & DAGVPLKEM & K203 \\
\hline HM_55378760 & EGTAA & S120 & RSGPDKAQISKDSLR & K171 \\
\hline HS_15789821 & RGPAA & S119 & KSAPDKGTTGPADKA & K166 \\
\hline HW_110667987 & TDTCA & S138 & YKDAGVPLREI & K190 \\
\hline $\mathrm{HW}_{-} 110668686$ & EGTAA & S122 & AQITRDGLR & K173 \\
\hline HW_110668977 & QGVSASGAY & S120 & QPTTEQRR & K165 \\
\hline MJ_15668832 & EGLDZ & S128 & DIGSPERPM & K180 \\
\hline MB_91773876 & GDVAA & S229 & DVGGDWRGL & K281 \\
\hline MMP_ 45358632 & ENMGA & S121 & KDIGTPTRSM & K173 \\
\hline MA_z̄20092840 & GDLAAS & S210 & MGSSWRGL & K262 \\
\hline MBA__73668088 & GDLA & S213 & MGSTWRGL & K265 \\
\hline MMZ_21226968 & GDLAAS & S225 & KSGEFKDMGGTWRGL & K277 \\
\hline MTH_15678828 & SDSGT & S114 & KAGEYKDMGADYRMI & K166 \\
\hline $\mathrm{NP} \_\overline{7} 6800828$ & TDACAS & S155 & EAGVPEDDL & K207 \\
\hline $\mathrm{NP}_{-} 76802382$ & DTMGASGAYLS & S117 & DKRPSTEQQR & K161 \\
\hline $\mathrm{PT}^{-} 48477283$ & EGIGASGAYW̄L & S76 & YKDINSPERHM & K128 \\
\hline PA_14520806 & SGYAYSGAYYI & S164 & KTGPYKDMGADWRGL & K216 \\
\hline PF_18977955 & SGYATSGGYYI & S160 & KTGPYKDMGADWRGL & K212 \\
\hline $\mathrm{PH}_{-} 14591350$ & SGYAYSGAYYI & S163 & KTGPYKDMGADWRKL & K215 \\
\hline TK_57641099 & GDIIASGGYYI & S162 & KTGKHKDMGAEWRDL & K214 \\
\hline TA_16081247 & QGICASGAYWI & S76 & KVGKYKDMLSSYREP & K128 \\
\hline TV_13540860 & LGICASGAYWI & S76 & KSGKYKDMTSPESEP & K128 \\
\hline \multicolumn{5}{|l|}{ Nanoarchaeota } \\
\hline NE 41615105 & AQYGTSASYWNI & S103 & SKGKYKEFSNPLLPL & K155 \\
\hline
\end{tabular}

were targeted for mutagenesis in SppA to determine those essential for enzymic activity. Ser ${ }^{162}$, believed to act as the nucleophilic residue, and Lys ${ }^{214}$, believed to be the general base, are both invariant in archaeal SPPs (Table 4). Strikingly, histidine and aspartic acid residues that comprise the catalytic triad of many serine proteases are not conserved. Sitedirected mutagenesis of various other amino acid residues in the T. kodakaraensis enzyme caused a reduction in activity and, though not essential or completely conserved, these residues (e.g. $\mathrm{Arg}^{221}$ ) may play a role in catalysis. The data obtained suggest that the catalytic centre comprises a Ser-Lys dyad and not the usual Ser-His-Asp catalytic triad found in the majority of serine proteases. The alignment data clearly indicate that likely all archaea SPPs function in the same way. The proposed catalytic serine is present also in the SPPs of $B$. subtilis and E. coli. While the B. subtilis SPP also possesses the conserved lysine as the general base, the enzyme in E. coli does not; it has been suggested that this enzyme uses different residues for catalysis (Matsumi et al., 2006).

\section{Concluding remarks}

While studies are still in their infancy, indications are that in regard to signal peptidases, archaea have their own unusual twists. Archaeal SPI more closely resembles its eukaryal counterpart, requiring an essential His for catalysis but with the archaeal modification of needing only one Asp. Archaeal TFPPs have a catalytic mechanism that resembles that of the corresponding bacterial enzymes but again with archaeal specifics. The archaeal enzymes lack the methylase activity commonly associated with most bacterial TFPPs and the substrates include flagellins and sugar-binding proteins that are unique to archaea. Studies on the archaeal SPP pioneer the field of prokaryotic signal peptide peptidase research. Site-directed mutagenesis and alignment data clearly show archaeal SPPs to have a Ser-Lys catalytic dyad mechanism and this information may translate to bacterial systems as well. Still to be resolved is how archaeal proteins with apparent SPII signal peptides are processed, since SPII is as yet unidentified in any archaeon. One must also be cognizant of the fact that current programs used to detect archaeal signal peptides are trained on bacterial and eukaryal databases; it is highly possible that some archaeal-specific signal peptides have been missed. It has already been noted that some archaea express proteins with unusual signal peptides (Eichler, 2000). Proteomic analysis of secreted proteins from Methanococcoides burtonii is a recent, rare 
example combining in vivo and in silico approaches to further refine our ability to recognize archaeal signal peptide sequences (Saunders et al., 2006) and in turn contribute to our knowledge of archaeal signal peptidases.

\section{Note added in proof}

Recently, Szabo et al. (J Bacteriol 2006 Nov 17; [Epub ahead of print] PMID: 17114255) identified, throughout the archaea, a variety of diverse proteins, including possible pilins, that have class III signal peptides, which are cleaved by a prepilin peptidase-like enzyme distinct from FlaK/PibD.

\section{References}

Albers, S.-V. \& Driessen, A. M. (2002). Signal peptides of secreted proteins of the archaeon Sulfolobus solfataricus: a genomic survey. Arch Microbiol 177, 209-216.

Albers, S.-V., Szabo, Z. \& Driessen, A. J. M. (2003). Archaeal homolog of bacterial type IV prepilin signal peptidases with broad substrate specificity. J Bacteriol 185, 3918-3925.

Albers, S.-V., Szabo, Z. \& Driessen, A. J. M. (2006). Protein secretion in the Archaea: multiple paths towards a unique cell surface. Nat Rev Microbiol 4, 537-547.

Bardy, S. L. \& Jarrell, K. F. (2002). FlaK of the archaeon Methanococcus maripaludis possesses preflagellin peptidase activity. FEMS Microbiol Lett 208, 53-59.

Bardy, S. L. \& Jarrell, K. F. (2003). Cleavage of preflagellins by an aspartic acid signal peptidase is essential for flagellation in the archaeon Methanococcus voltae. Mol Microbiol 50, 1339-1347.

Bardy, S. L., Eichler, J. \& Jarrell, K. F. (2003). Archaeal signal peptides - a comparative survey at the genome level. Protein Sci 12, 1833-1843.

Bardy, S. L., Ng, S. Y., Carnegie, D. S. \& Jarrell, K. F. (2005). Sitedirected mutagenesis analysis of amino acids critical for activity of the type I signal peptidase of the archaeon Methanococcus voltae. J Bacteriol 187, 1188-1191.

Berks, B. C. (1996). A common export pathway for proteins binding complex redox cofactors? Mol Microbiol 22, 393-404.

Bolhuis, A. (2002). Protein transport in the halophilic archaeon Halobacterium sp. NRC-1: a major role for the twin-arginine translocation pathway? Microbiology 148, 3335-3346.

Bolhuis, A., Matzen, A., Hyyrylainen, H.-L., Kontinen, V. P., Meimer, R., Chapuis, J., Venema, G., Bron, S., Freudl, R. \& van Dijl, J. M. (1999). Signal peptide peptidase- and ClpP-like proteins of Bacillus subtilis required for efficient translocation and processing of secretory proteins. J Biol Chem 274, 24585-24592.

Chaban, B., Ng, S. Y. \& Jarrell, K. F. (2006). Archaeal habitats - from the extreme to the ordinary. Can J Microbiol 52, 73-116.

Dalbey, R. E., Lively, M. O., Bron, S. \& van Dijl, J. M. (1997). The chemistry and enzymology of the type I signal peptidases. Protein Sci 6, 1129-1138.

Eichler, J. (2000). Archaeal protein translocation: crossing membranes in the third domain of life. Eur J Biochem 267, 3402-3412.

Eichler, J. (2002). Archaeal signal peptidases from the genus Thermoplasma: structural and mechanistic hybrids of the bacterial and eukaryal enzymes. J Mol Evol 54, 411-415.

Falb, M., Aivaliotis, M., Garcia-Rizo, C., Tebbe, B. B. A., Klein, C., Konstantinidis, K., Siedler, F., Pfeiffer, F. \& Oesterhelt, D. (2006). Archaeal $\mathrm{N}$-terminal protein maturation commonly involves $\mathrm{N}$ terminal acetylation: a large-scale proteomics survey. J Mol Biol 262, 915-924.
Fine, A., Irihimovitch, V., Dahan, I., Konrad, Z. \& Eichler, J. (2006). Cloning, expression, and purification of functional Sec1la and Sec11b, type I signal peptidases of the archaeon Haloferax volcanii. J Bacteriol 188, 1911-1919.

LaPointe, C. F. \& Taylor, R. K. (2000). The type IV prepilin peptidases comprise a novel family of aspartic acid proteases. J Biol Chem 275, 1502-1510.

Lemberg, M. K. \& Martoglio, B. (2004). On the mechanism of SPPcatalysed intramembrane proteolysis: conformational control of peptide bond hydrolysis in the plane of the membrane. FEMS Lett 564, 213-218.

Lory, S. \& Strom, M. S. (1997). Structure-function relationship of type-IV prepilin peptidase of Pseudomonas aeruginosa - a review. Gene 192, 117-121.

Matsumi, R., Atomi, H. \& Imanaka, T. (2005). Biochemical properties of a putative signal peptide peptidase from the hyperthermophilic archaeon Thermococcus kodakaraensis KOD1. J Bacteriol 187, 7072-7080.

Matsumi, R., Atomi, H. \& Imanaka, T. (2006). Identification of the amino acid residue essential for proteolytic activity in an archaeal signal peptide peptidase. J Biol Chem 281, 10533-10539.

Mattick, J. S. (2002). Type IV pili and twitching motility. Annu Rev Microbiol 56, 289-314.

Ng, S. Y. M. \& Jarrell, K. F. (2003). Cloning and characterization of archaeal type I signal peptidase from Methanococcus voltae. J Bacteriol 185, 5936-5942.

Ng, S. Y. M., Chaban, B. \& Jarrell, K. F. (2006). Archaeal flagella, bacterial flagella and type IV pili: a comparison of genes and posttranslational modifications. J Mol Microbiol Biotechnol 11, 167-191.

Nielsen, H., Brunak, S. \& von Heijne, G. (1999). Machine learning approaches for the prediction of signal peptides and other learning sorting signals. Protein Eng 12, 3-9.

Novak, P. \& Dev, I. K. (1998). Degradation of a signal peptide by protease IV and oligopeptidase A. J Bacteriol 170, 5065-5075.

Paetzel, M., Dalbey, R. E. \& Strynadka, N. C. J. (2002). Crystal structure of a bacterial signal peptidase apoenzyme. J Biol Chem 277, 9512-9519.

Pohlschroder, M., Prinz, W. A., Hartmann, E. \& Beckwith, J. (1997). Protein translocation in the three domains of life: variations on a theme. Cell 91, 563-566.

Pohlschroder, M., Gimenez, M. I. \& Jarrell, K. F. (2005). Protein transport in Archaea: Sec and twin arginine translocation pathways. Curr Opin Microbiol 8, 713-719.

Rose, R. W., Bruser, T., Kissinger, J. C. \& Pohlschroder, M. (2002). Adaptation of protein secretion to extremely high-salt conditions by extensive use of the twin-arginine translocation pathway. Mol Microbiol 45, 943-950.

Saleh, M. T., Fillon, M., Brennan, P. J. \& Belisle, J. T. (2001). Identification of putative exported/secreted proteins in prokaryotic proteomes. Gene 269, 195-204.

Sargent, F., Berks, B. C. \& Palmer, T. (2006). Pathfinders and trailblazers: a prokaryotic targeting system for transport of folded proteins. FEMS Microbiol Lett 254, 198-207.

Saunders, S. F., Ng, C., Raftery, M., Guihaus, M., Goodchild, A. \& Cavicchioli, R. (2006). Proteomic and computational analysis of secreted proteins with type I signal peptides from the Antarctic archaeon Methanococcoides burtonii. J Proteome Res 5, 2457-2464.

Steiner, H. \& Haass, C. (2000). Intramembrane proteolysis by presenilins. Nat Rev Mol Cell Biol 1, 217-224. 
Szabo, Z., Albers, S.-V. \& Driessen, A. J. M. (2006). Active-site residues in the type IV prepilin peptidase homologue PibD from the archaeon Sulfolobus solfataricus. J Bacteriol 188, 1437-1443.

Thomas, N. A., Chao, E. D. \& Jarrell, K. F. (2001). Identification of amino acids in the leader peptide of Methanococcus voltae preflagellin that are important in posttranslational processing. Arch Microbiol $175,263-269$.

Tjalsma, H., van den Dolder, J., Meijer, W. J., Venema, G., Bron, S. \& van Dijl, J. M. (1999). The plasmid-encoded signal peptidase SipP can functionally replace the major signal peptidases SipS and SipT of Bacillus subtilis. J Bacteriol 181, 2448-2454.

Tjalsma, H., Stover, A. G., Driks, A., Venema, G., Bron, S. \& van Dijl, J. M. (2000). Conserved serine and histidine residues are critical for activity of the ER-type signal peptidase SipW of Bacillus subtilis. J Biol Chem 275, 25102-25108.

Tomich, M., Fine, D. H. \& Figurski, D. H. (2006). The TadV protein of Actinobacillus actinomycetemcomitans is a novel aspartic acid prepilin peptidase required for maturation of the Flp1 pilin and TadE and TadF pseudopilins. J Bacteriol 188, 6899-6914.
Tschantz, W. R., Sung, M., Dalgado-Partin, V. M. \& Dalbey, R. E. (1993). A serine and a lysine residue implicated in the catalytic mechanism of Escherichia coli leader peptidase. J Biol Chem 268, 27349-27354.

Tuteja, R. (2005). Type I signal peptidase: an overview. Arch Biochem Biophys 441, 107-111.

VanValkenburgh, C., Chen, X., Mullins, C., Fang, H. \& Green, N. (1999). The catalytic mechanism of endoplasmic reticulum signal peptidase appears to be distinct from most eubacterial signal peptidases. J Biol Chem 274, 11519-11525.

von Heijne, G. (1983). Patterns of amino acids near signal sequence cleavage sites. Eur J Biochem 133, 17-21.

Woese, C. R., Kandler, O. \& Wheelis, M. L. (1990). Towards a natural system of organisms: proposal for the domains Archaea, Bacteria, and Eucarya. Prot Natl Acad Sci U S A 87, 4576-4579.

Yamasaki, A., Eimer, S., Okochi, M., Smialowska, A., Kaether, C., Baumeister, R., Haass, C. \& Steiner, H. (2006). The GxGD motif of presenilin contributes to catalytic function and substrate identification of $\gamma$-secretase. J Neurosci 26, 3821-3828. 